\title{
Field-enhanced route to generating anti-Frenkel pairs in $\mathrm{HfO}_{2}$
}

\author{
Marcel Schie* \\ Institute of Materials in Electrical Engineering and Information Technology II, RWTH Aachen University, 52056 Aachen, Germany \\ Stephan Menzel \\ Peter Grünberg Institute (PGI-7), Research Centre Jülich, 52425 Jülich, Germany \\ John Robertson \\ Department of Engineering, University of Cambridge, Cambridge CB3 OFA, United Kingdom \\ Rainer Waser \\ Institute of Materials in Electrical Engineering and Information Technology II, RWTH Aachen University, 52056 Aachen, Germany \\ and Peter Grünberg Institute (PGI-7), Research Centre Jülich, 52425 Jülich, Germany \\ Roger A. De Souza ${ }^{\dagger}$ \\ Institute of Physical Chemistry, RWTH Aachen University and JARA-FIT, 52056 Aachen, Germany
}

(Received 22 December 2017; published 19 March 2018)

\begin{abstract}
The generation of anti-Frenkel pairs (oxygen vacancies and oxygen interstitials) in monoclinic and cubic $\mathrm{HfO}_{2}$ under an applied electric field is examined. A thermodynamic model is used to derive an expression for the critical field strength required to generate an anti-Frenkel pair. The critical field strength of $\mathcal{E}_{\mathrm{aF}}^{\mathrm{cr}} \sim 10^{1} \mathrm{GVm}^{-1}$ obtained for $\mathrm{HfO}_{2}$ exceeds substantially the field strengths routinely employed in the forming and switching operations of resistive switching $\mathrm{HfO}_{2}$ devices, suggesting that field-enhanced defect generation is negligible. Atomistic simulations with molecular static (MS) and molecular dynamic (MD) approaches support this finding. The MS calculations indicated a high formation energy of $\Delta E_{\mathrm{aF}} \approx 8 \mathrm{eV}$ for the infinitely separated anti-Frenkel pair, and only a decrease to $\Delta E_{\mathrm{aF}} \approx 6 \mathrm{eV}$ for the adjacent anti-Frenkel pair. The MD simulations showed no defect generation in either phase for $\mathcal{E}<3 \mathrm{GVm}^{-1}$, and only sporadic defect generation in the monoclinic phase (at $\mathcal{E}=3 \mathrm{GVm}^{-1}$ ) with fast $\left(t_{\mathrm{rec}}<4 \mathrm{ps}\right.$ ) recombination. At even higher $\mathcal{E}$ but below $\mathcal{E}_{\mathrm{aF}}^{\mathrm{cr}}$ both monoclinic and cubic structures became unstable as a result of field-induced deformation of the ionic potential wells. Further MD investigations starting with preexisting anti-Frenkel pairs revealed recombination of all pairs within $t_{\text {rec }}<1 \mathrm{ps,}$ even for the case of neutral vacancies and charged interstitials, for which formally there is no electrostatic attraction between the defects. In conclusion, we find no physically reasonable route to generating point-defects in $\mathrm{HfO}_{2}$ by an applied field.
\end{abstract}

DOI: 10.1103/PhysRevMaterials.2.035002

\section{INTRODUCTION}

Point defects are unavoidable. They are an equilibrium component of all crystals at finite temperature. As a consequence, their concentrations can be predicted from thermodynamics for the system's given degrees of freedom. These degrees of freedom are temperature, the type and amount of purposely added dopants (or inadvertently included impurities), and in compounds, the relevant component chemical potentials. Modifying the concentrations of point defects in a crystal can be accomplished, therefore, by thermal means (varying the temperature) and by chemical means (varying the type and the concentration of dopants or varying the appropriate chemical potentials) [1-6].

It is possible to achieve point-defect concentrations beyond their equilibrium values; the most well-known of these

\footnotetext{
*schie@iwe.rwth-aachen.de

†desouza@pc.rwth-aachen.de
}

nonequilibrium mechanisms being irradiation [6-9]. The passage of high-energy particles through a lattice displaces ions from their regular sites generating vacancies and interstitials; many of these defects will recombine over a relatively short time scale, but, depending on the kinetics of the recombination process, a significant fraction may remain in the material.

Recently, a different nonequilibrium mechanism for generating point defects has been proposed and has received increasing attention: the application of an electric field to produce vacancy-interstitial pairs. In particular, this mechanism is said to play a central role in two separate phenomena: resistive switching [10,11] and flash sintering [12,13]. More generally, one may expect field-induced defect generation to play a role wherever the electric field strength is high, that is, in nanoscaled (electrochemical $[14,15]$, photovoltaic, or ferroelectric $[16,17])$ devices and at interfaces with spacecharge layers [18-20]. In this study, we take crystalline $\mathrm{HfO}_{2}$, a promising candidate material for resistive switching devices, as a model system to study the possibility of field-induced generation of oxygen vacancies and oxygen interstitials. 
Electron-hole pair

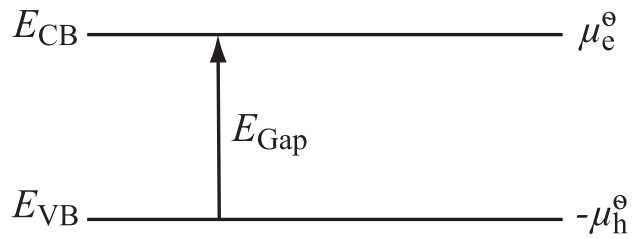

Anti-Frenkel pair

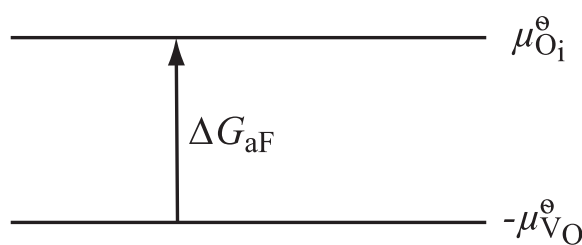

FIG. 1. Thermodynamic analogy of electron-hole and and interstitial-vacancy formation. $\mu_{j}^{o}$ is the standard chemical potential of defect $j$.

The phenomenon of resistive switching is known in many materials, including $\mathrm{HfO}_{2}$, to involve the formation of an electrically conducting filament [21,22]. This process can be separated into two mechanistic steps: (1) the formation of oxygen vacancies and (2) their aggregation to form a conducting filament. For the first step, two opposing models have emerged. One is based on the formation of doubly positively charged oxygen vacancies $\left(\mathrm{V}_{\mathrm{O}}^{* \bullet}\right)$ near an interface according to the following reduction reaction

$$
\mathrm{O}_{\mathrm{O}}^{\times} \rightarrow \ddot{\mathrm{V}_{\mathrm{O}}}+2 \mathrm{e}^{\prime}+\mathrm{O}_{\text {ext }} .
$$

The charged vacancies migrate in the oxide layer under the applied electric field to form the filament. The oxygen atoms $\mathrm{O}_{\text {ext }}$ are either incorporated into an oxygen reservoir (the oxygen exchange layer) or, after recombination with a second oxygen atom, desorbed from the surface as an oxygen molecule.

The alternative model relies on the hypothesis that oxygen vacancies and oxygen interstitials (anti-Frenkel pairs [1]) can be generated by an electric field $\mathcal{E}$ [23-32]:

$$
\mathrm{O}_{\mathrm{O}}^{\times} \stackrel{\mathcal{E}}{\rightarrow} \mathrm{V}_{\mathrm{O}}^{\ddot{*}}+\mathrm{O}_{\mathrm{i}}^{\prime \prime},
$$

that is, oxygen ions are forced to move from their regular lattice sites to sit on the interstitial sublattice, leaving a vacant site behind. The recombination of interstitial and vacancy species, though favorable owing to the strong Coulomb interaction between the oppositely charged defects, is prevented by a second process, the (fast) localization of two electrons $\left(2 \mathrm{e}^{\prime}\right)$ at the newly formed vacancy, $\mathrm{V}_{\mathrm{O}}^{\cdot \bullet}$, to form a neutral vacancy, $\mathrm{V}_{\mathrm{O}}^{\times}$. (Note: the two electrons have been proposed to localize either at the nearest-neighbor Hf ions $[33,34]$, or at the vacant site itself $[31,35,36]$.) The oxygen interstitial is now free to migrate under the external electric field. In a further variant of this mechanism [37,38], the formation energy of anti-Frenkel pairs is considered to be lowered in the direct vicinity of a double negatively charged oxygen vacancy:

$$
\mathrm{O}_{\mathrm{O}}^{\times}+\mathrm{V}_{\mathrm{O}}^{\prime \prime} \stackrel{\mathcal{E}}{\rightarrow} 2 \mathrm{~V}_{\mathrm{O}}^{\times}+\mathrm{O}_{\mathrm{i}}^{\prime \prime} .
$$

This proposal thus requires a bare oxygen vacancy to trap four electrons, forming a moiety $\left(\mathrm{V}_{\mathrm{O}}^{\prime \prime}\right)$ that allows the newly formed oxygen vacancy $\left(\mathrm{V}_{\mathrm{O}}^{\bullet \bullet}\right)$ to be neutralized immediately. Both neutral vacancies subsequently trap further electrons to continue the formation of anti-Frenkel pairs and, in this way, form a conducting filament consisting of oxygen vacancies $[37,38]$.

This study is concerned with the generation and recombination of anti-Frenkel pairs in $\mathrm{HfO}_{2}$ under an applied electric field. To this end, we construct a (simple) thermodynamic model to estimate a critical value of the field required to create anti-Frenkel pairs. Subsequently, we employ molecular static (MS) and molecular dynamics (MD) simulations using empirical pair-potentials (EPP) to examine point-defect processes in $\mathrm{HfO}_{2}$. In particular, we calculate the formation energies of an anti-Frenkel pair as a function of defect-defect separation in MS simulations, and we examine the generation and the recombination of anti-Frenkel pairs under applied fields in MD simulations.

\section{A SIMPLE THERMODYNAMIC MODEL}

There is a close analogy between the thermodynamics of electron-hole formation in a semiconductor and interstitialvacancy formation in an ionic crystal. In both cases, a regular particle is excited across an energy gap to generate an excess particle (electron, interstitial) and a missing particle (hole, vacancy) $[39,40]$. In the case of electrons in a semiconductor, this energy is termed the band gap $E_{\mathrm{g}}$; in the case of oxygen ions in a crystalline oxide, it is the Gibbs energy of anti-Frenkel disorder $\Delta G_{\mathrm{aF}}$. The close analogy is emphasized in the relevant energy-band diagrams shown in Fig. 1.

A further commonality is that, in each case, the product of the particles' concentrations is equal to an equilibrium constant that shows a simple exponential dependence on the relevant energy, as long as dilute-solution thermodynamics holds. For the electronic case, i.e., the product of the equilibrium concentrations of electrons and holes, one has

$$
\left[\mathrm{e}^{\prime}\right]\left[\mathrm{h}^{\bullet}\right]=K_{\mathrm{eh}}(T)=N_{\mathrm{CB}}(T) N_{\mathrm{VB}}(T) \exp \left(-\frac{E_{\mathrm{g}}(T)}{k_{\mathrm{B}} T}\right),
$$

where $N_{\mathrm{CB}}(T)$ and $N_{\mathrm{VB}}(T)$ are the temperature-dependent densities of states at the conduction-band (CB) and valenceband (VB) edges. Doping with a sufficient amount of a donor, for example, increases $\left[\mathrm{e}^{\prime}\right]$, and through Eq. (4), decreases $\left[\mathrm{h}^{\bullet}\right]$. For the ionic case, one obtains that the product of the equilibrium concentrations is given by

$$
\left[\mathrm{O}_{\mathrm{i}}^{\prime \prime}\right]\left[\mathrm{V}_{\mathrm{O}}^{\bullet \bullet}\right]=K_{\mathrm{iv}}(T)=N_{\mathrm{i}} N_{\mathrm{v}} \exp \left(-\frac{\Delta G_{\mathrm{aF}}(T)}{k_{\mathrm{B}} T}\right),
$$

where $N_{\mathrm{i}}$ and $N_{\mathrm{v}}$ are the temperature-independent densities of sites in the interstitial (i) and vacancy (v) sublattices. Doping with an acceptor, say, raises [ $\mathrm{V}_{\mathrm{O}}^{\bullet \bullet}$, and through Eq. (5), diminishes $\left[\mathrm{O}_{\mathrm{i}}^{\prime \prime}\right]$. It is emphasized that in a semiconducting oxide, such as $\mathrm{HfO}_{2}$, these two processes are linked: By reducing an oxide according to Eq. (1), the concentrations of 

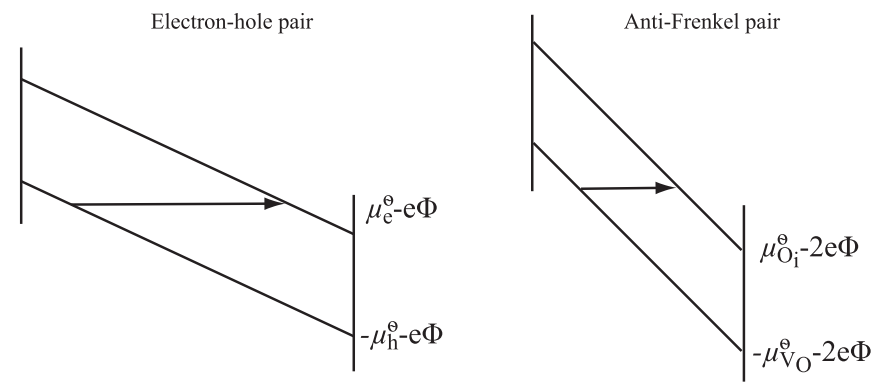

FIG. 2. Thermodynamic analogy of electron-hole and interstitialvacancy generation under an electric field.

oxygen vacancies and electrons will be increased, and those of oxygen interstitials and electron holes will be decreased.

The application of an electric field to a semiconductor or an oxide crystal results in both cases in the energy levels becoming tilted (see Fig. 2), albeit with different gradients, $e \mathcal{E}$ for the (singly charged) electrons versus $2 e \mathcal{E}$ for the (doubly charged) oxygen ions. The field-enhanced generation of defects corresponds generally, then, to the particle (electron, ion) traversing the relevant energy gap, from an occupied state/site to an unoccupied state/site (electrons, from VB to $\mathrm{CB}$; ions, from regular to interstitial sublattice), by moving a distance $d$ in the field $\mathcal{E}$. In other words, the energy required to bridge the relevant energy gap is supplied by the electric field, and for a particle of charge ze, this energy is given by $z e \mathcal{E} d$. In this way, one can estimate the critical field strength $\mathcal{E}^{\text {cr }}$ required for the generation of $\mathrm{e}^{\prime}-\mathrm{h}^{\bullet}$ or $\mathrm{O}_{\mathrm{i}}^{\prime \prime}-\mathrm{V}_{\mathrm{O}}^{\bullet \bullet}$ pairs.

Besides all the commonalities there is a fundamental difference between the two cases. Electrons can quantum mechanically tunnel from the valence band to the conduction band-the Zener effect [41]. Tunnelling is not feasible in the ionic case, however, because ions have a prohibitively high mass. Consequently, the characteristic distances $d$ differ hugely: electrons can tunnel up to $d_{\mathrm{tnl}} \approx 10^{2} \AA$ [42], whereas oxygen ions, in jumping from a regular oxygen site to a neighboring interstitial site, necessarily move distances $\left(d_{\mathrm{vi}}\right)$ that are only a few $\AA$.

Consequently, for the field-induced generation of antiFrenkel pairs in $\mathrm{HfO}_{2}$, we can write

$$
\mathcal{E}_{\mathrm{aF}}^{\mathrm{cr}}=\frac{\Delta G_{\mathrm{aF}}}{2 e d_{\mathrm{vi}}}
$$

and taking $d_{\mathrm{vi}}=2.5 \AA[38,43]$ and assuming $\Delta G_{\mathrm{aF}} \approx$ $\Delta H_{\mathrm{aF}}=(\min ; \max )=(4.7 \mathrm{eV} ; 8.0 \mathrm{eV}) \quad[31,36,44,45]$, we find a critical field strength $\mathcal{E}_{\mathrm{aF}}^{\mathrm{cr}}=(\min ; \max )=\left(9.4 \mathrm{GVm}^{-1}\right.$; $\left.16.0 \mathrm{GVm}^{-1}\right)$. Other fluorite-structured oxides are predicted to exhibit similar values of $\Delta H_{\mathrm{aF}}\left(\mathrm{CeO}_{2}:(4.1 \mathrm{eV}\right.$; $6.4 \mathrm{eV})$ [46,47]; $\mathrm{UO}_{2}:(4.8 \mathrm{eV} ; 5.7 \mathrm{eV})$ [47-49]; $\mathrm{ThO}_{2}$ : $(5.0 \mathrm{eV} ; 6.8 \mathrm{eV})[47,50,51])$ and thus will be characterized by similar critical field strengths. The equivalent expression for electron-hole generation in Si gives a critical field strength of $\mathcal{E}_{\mathrm{eh}}^{\mathrm{cr}}=E_{\mathrm{g}} /\left(e d_{\mathrm{tnl}}\right) \sim 10^{-1} \mathrm{GV} \mathrm{m}^{-1}$, in good agreement with experimental data for reverse-biased $p-n$ junctions [42].

Thus, according to this simple treatment, the critical field strength required for anti-Frenkel generation in $\mathrm{HfO}_{2}$ is enormous. For a resistive-switching cell containing a 5-nm-thick $\mathrm{HfO}_{2}$ layer, Eq. (6) indicates that an applied voltage of
$(47 \mathrm{~V} ; 80 \mathrm{~V})$ would be necessary to form or switch the cell, whereas in reality such cells can easily be formed or switched with voltages of $3 \mathrm{~V}$ or lower [52-56]. Furthermore, it is well known that significantly thicker films (up to 40-nm thick) can be switched with voltages less than $5 \mathrm{~V}$ [57-59], in contrast to the $(376 \mathrm{~V} ; 640 \mathrm{~V})$ that would be needed for field-enhanced pair formation according to Eq. (6). The fields even exceed the breakdown field of the best insulators (e.g., $\mathrm{SiO}_{2}$ ), which is approximately $1 \mathrm{GV} \mathrm{m}^{-1}$ [60]. This treatment thus suggests that field-induced generation of anti-Frenkel pairs is not an important process in filament formation.

There are four effects, not included in the treatment above, that could be expected to modify the critical field in some manner: entropy, other charge states, finite dilution, and kinetics. From the discussion above, it is clear that, either on their own or in combination, these effects have to decrease $\Delta G_{\mathrm{aF}}$ by roughly one order of magnitude for field-induced pair formation to become important.

First, the entropy of anti-Frenkel formation $\Delta S_{\mathrm{aF}}$ needs to be included because $\Delta G_{\mathrm{aF}}\left(=\Delta H_{\mathrm{aF}}-T \Delta S_{\mathrm{aF}}\right)$ is required, whereas we use $\Delta H_{\mathrm{aF}} . \Delta S_{\mathrm{aF}}$ is not known for $\mathrm{HfO}_{2}$, but Grieshammer et al. [61] obtained for structurally similar (cubic fluorite-structured) $\mathrm{CeO}_{2}$, by means of density functional theory (DFT) calculations, a value of $\Delta S_{\mathrm{aF}}=10 k_{\mathrm{B}}$. This means that at $T=300 \mathrm{~K}$ the entropic contribution to $\Delta G_{\mathrm{aF}}$ is only $-T \Delta S_{\mathrm{aF}}=-0.26 \mathrm{eV}$. The inclusion of entropy, on its own, will not, therefore, reduce the critical field substantially.

Second, the values of $\Delta H_{\mathrm{aF}}$ we use refer to $\mathrm{V}_{\mathrm{O}}^{*}$ and $\mathrm{O}_{\mathrm{i}}^{\prime \prime}$, but these defects may of course assume other charge states. Zheng et al. [44], however, reported that the formation of a pair of singly charged defects $\left(\mathrm{V}_{\mathrm{O}}^{*}, \mathrm{O}_{\mathrm{i}}^{\prime}\right)$ in $\mathrm{HfO}_{2}$ has an even higher formation energy than that of the pair of doubly charged defects, and that the formation energy is higher still for a pair of neutral defects $\left(\mathrm{V}_{\mathrm{O}}^{\times}, \mathrm{O}_{\mathrm{i}}^{\times}\right)$. Similar results have also been reported for anti-Frenkel pairs in cubic fluorite-structured $\mathrm{ThO}_{2}$ [50,51] and $\mathrm{UO}_{2}$ [49]. This effect can, therefore, be safely ignored.

Third, in all literature studies $[31,36,44,45]$ the values of $\Delta H_{\mathrm{aF}}$ refer to infinite dilution (and thus to infinite separation) of the anti-Frenkel pair. Immediately after the oxygen ion has left its site to take up an interstitial position, however, the vacancy and interstitial species are separated by only a few $\AA$, and this effect of finite dilution (or equivalently, of finite separation) may serve to lower $\Delta H_{\mathrm{aF}}$. Instead of Eq. (2), we write, therefore,

$$
\mathrm{O}_{\mathrm{O}}^{\times} \stackrel{\mathcal{E}}{\rightarrow}\left\{\mathrm{V}_{\mathrm{O}}^{\ddot{*}}-\mathrm{O}_{\mathrm{i}}^{\prime \prime}\right\} \stackrel{\mathcal{E}}{\rightarrow} \mathrm{V}_{\mathrm{O}}^{\ddot{*}}+\mathrm{O}_{\mathrm{i}}^{\prime \prime}
$$

The first process refers to the formation of a bound vacancyinterstitial pair (the electronic equivalent is the exciton); the second process, to the dissociation of the pair. A simple calculation based solely on the electrostatic potential energy of the defect pair (with $\epsilon_{\mathrm{r}}=25$ ) gives a decrease in formation energy of the pair by ca. $1 \mathrm{eV}$ (as long as the pair are never separated by an infinite distance). This estimation neglects, however, elastic interactions between the defects and also the local relaxations of ions around the defects; it also uses the macroscopic relative dielectric permittivity at a scale where the discrete nature of the lattice may be important. This 
effect requires closer examination (and we use static atomistic simulations to examine it, as described in Sec. IV A).

Fourth, the model, being a thermodynamic model, does not include any kinetic effects. It ignores that the oxygen ion, on moving from its regular site to the interstitial site [the first process of Eq. (7)], may have to pass over an activation barrier $\left(\Delta E_{\mathrm{aFm}}\right)$. That is, there is a maximum in the energy landscape between the oxygen ion sitting on its regular site and having moved to the interstitial lattice. This barrier would effectively increase the critical field required to generate the anti-Frenkel pair, but it would also hinder recombination of interstitial and vacancy. An added complication is that this barrier, if it exists, will be modified by an applied field [62]. While Walsh et al. [63] reported an energy difference of $\Delta E_{\mathrm{aF}}+\Delta E_{\mathrm{aFm}}=$ $5.02+0.78 \mathrm{eV}$ for the oxygen ion moving to the interstitial site in $\mathrm{CeO}_{2}$, Traore et al. [31] found no activation barrier for this process in $\mathrm{HfO}_{2}$, i.e., an energy difference of only $\Delta E_{\mathrm{aF}}$. In addition, Clima et al. [64] in MD simulations observed immediate recombination of anti-Frenkel pairs, a result that supports this interpretation as it implies, as noted by Clima et al. [64], that there is no activation barrier.

A more important kinetic issue is the increase in the equilibrium constant of the dissociation reaction [the second process of Eq. (7)] by an electric field. This effect was examined in detail (for ion pairs in solution) by Onsager in 1934 [65], and it was later applied to exciton pairs in semiconductors by Braun [66]. In Onsager's treatment, the rate of dissociation $\left(k_{\mathrm{d}}\right)$ is enhanced by the field, whereas the rate of association $\left(k_{\mathrm{a}}\right)$ is unaffected. This leads to the equilibrium constant for dissociation $\left(K_{\mathrm{d}}=k_{\mathrm{d}} / k_{\mathrm{a}}\right)$ being shifted in a field according to

$$
K_{\mathrm{d}}(\mathcal{E}, T)=K_{\mathrm{d}}(0, T)\left(1+\beta+\frac{\beta^{2}}{3}+\frac{\beta^{3}}{18}+\mathcal{O}\left(\beta^{4}\right)+\ldots\right)
$$

with

$$
\beta=\frac{(z e)^{3} \mathcal{E}}{8 \pi \epsilon_{0} \epsilon_{\mathrm{r}}\left(k_{\mathrm{B}} T\right)^{2}} .
$$

In the case of $\left\{\mathrm{O}_{\mathrm{i}}^{\prime \prime}-\mathrm{V}_{\mathrm{O}}^{\bullet \bullet}\right\}$ dissociation in $\mathrm{HfO}_{2}$, the change in $K_{\mathrm{d}}(\mathcal{E}, T)$ at $\mathcal{E}=1 \mathrm{GV} \mathrm{m}^{-1}$, according to Eq. (8), results in a decrease in $\Delta G\left(=-R T \ln K_{\mathrm{d}}\right)$ of only $0.4 \mathrm{eV}$ (and the vacancy and interstitial have to be separated infinitely).

Thus, the inclusion of kinetic effects does not appear to change the overall picture. To support these conclusions concerning kinetic issues, we perform molecular dynamic (MD) atomistic simulations using classical empirical pair potentials (EPP), with and without an applied field (see Secs. IV B and IV C).

\section{COMPUTATIONAL METHODOLOGY}

The MS and MD simulations in this study are based on the Born model of polar solids. All ions bear their formal charges, e.g., $\mathrm{O}^{2-}$ and $\mathrm{Hf}^{4+}$, and they interact with each other through long-range Coulomb interactions and shortrange interactions. The short-range interactions are represented by parametrized Buckingham - two-body, spherically symmetrical-potentials of the form $\phi=A \cdot \exp ^{(-r / \rho)}-\frac{C}{r^{6}}$. We use the set of empirical potentials derived by Lewis and Catlow (see Table I). We have shown previously that these potentials are able to model oxygen-ion transport in cubic and
TABLE I. Parameters for the empirical Buckingham potential by Lewis and Catlow [71].

\begin{tabular}{lrrrr}
\hline \hline & \multicolumn{1}{c}{$A / \mathrm{eV}$} & $\rho / \AA$ & $C / \mathrm{eV} \cdot \AA^{6}$ & $z / \mathrm{e}$ \\
\hline $\mathrm{Hf}^{4+}-\mathrm{O}^{2-}$ & 1454.6 & 0.3500 & 0.000 & +4.0 \\
$\mathrm{O}^{2-}-\mathrm{O}^{2-}$ & 22764.3 & 0.1490 & 27.879 & -2.0 \\
\hline \hline
\end{tabular}

monoclinic (and also amorphous) $\mathrm{HfO}_{2}$ [67,68]. Initial cell vectors and ion positions were taken from Jaffe et al. [69] and were relaxed with the GULP [70] code to the minimum cell energy.

The MD simulations employed the Velocity-Verlet algorithm [72] to propagate the system through phase space and the thermostat and barostat by Martyna et al. [73] to establish a $N p T$ ensemble (constant particle number $N$, pressure $p$ and temperature $T$ ). The DL_POLY code [74] was used for all MD simulations. $10 a \times 10 a \times 10 a$ supercells with 12000 ions, for the stoichiometric state, and 11950 ions, for the reduced state were generated. The surplus charge was compensated by a homogeneous background charge (this has been shown for vacancy dynamics in $\mathrm{SrTiO}_{3}$ [75] to be a physically reasonable procedure).

\section{ATOMISTIC SIMULATIONS}

\section{A. Formation energy of anti-Frenkel pairs}

The formation energy of anti-Frenkel pairs in $\mathrm{HfO}_{2}$ as a function of vacancy-interstitial separation was studied with MS atomistic simulations based on the Mott-Littleton approach [76]. For the infinitely separated pair (two separate calculations), formation energies of around $8 \mathrm{eV}$ are found for both the cubic phase and for the monoclinic phase (with the 3-fold coordinated vacancy), in agreement with the DFT calculations by Foster et al. [36] and Guo and Robertson [45]. Significantly lower energy values, around $5 \mathrm{eV}$, have also been reported [44], but these studies do not correct for the finite size of the DFT-simulation cell.

Figure 3 shows the formation energy of an anti-Frenkel pair for the defect-defect distances between 2.2 and $28 \AA$ achievable with the Mott-Littleton approach. One sees that $\Delta E_{\mathrm{aF}}$ decreases substantially with decreasing separation. Also plotted is the electrostatic potential energy calculated, as before, with $\epsilon_{\mathrm{r}}=25$ and assuming point charges of $+2 \mathrm{e}$ and $-2 \mathrm{e}$. The comparison shows that not only electrostatic but also elastic contributions, as well as local ion relaxations, serve to bring about this reduction in energy. Nevertheless, the decrease in formation energy is only $2 \mathrm{eV}$, and consequently, the formation energy of an adjacent anti-Frenkel pair remains high at $\Delta E_{\mathrm{aF}} \approx 6 \mathrm{eV}$.

\section{B. Dynamics of anti-Frenkel pair formation}

MD simulations were performed to examine the dynamics of anti-Frenkel pair generation by an electric field. Such simulations were carried out for both monoclinic and cubic stoichiometric $\mathrm{HfO}_{2}$ at field strengths in the range $0<\mathcal{E} / \mathrm{GVm}^{-1}<5$ at $T=1000 \mathrm{~K}$ for $t_{\text {sim }}=1 \mathrm{~ns}$, and ion positions were tracked every picosecond. The high temperature was chosen to increase 


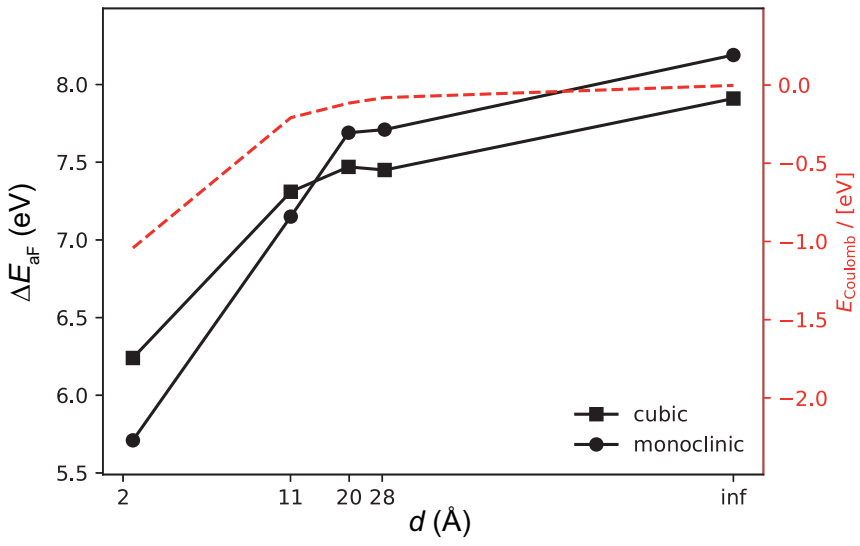

FIG. 3. Molecular static (MS) atomistic calculations of the formation energy of an anti-Frenkel pair in the cubic and monoclinic phases of $\mathrm{HfO}_{2}$. For the calculations at finite separation the defects were separated along the $\langle 111\rangle$ direction. The red dashed line shows a simple calculation of the Coulomb contribution to the energies.

significantly the likelihood of observing pair formation. We detected if an anti-Frenkel pair had formed by monitoring the distance between an oxygen ion's initial position and its position at time $t$. If this distance was larger than $1.25 \AA$, that is half the equilibrium separation of oxygen ions, then a Frenkel pair was considered to have formed. Once identified, such configurations were examined individually in detail.

The evaluation of the simulations yielded two particular results. First, for fields of $\mathcal{E}=4 \mathrm{GVm}^{-1}$ and larger, the structure for both the cubic and the monoclinic supercell became unstable, resulting in all ions drifting continuously. This behavior, as reported by Genreith-Schriever and De Souza [62], is due to the field deforming the potential well in which the oxygen ions sit so severely that in one direction there is no longer any confining barrier. The field at which this occurs can be calculated, given the height of the migration barrier in the field-free case $\left(\Delta E_{\mathrm{m}}\right)$. The calculations give $\mathcal{E}_{\mathrm{m}, \text { cub }}^{\mathrm{cr}}=$ $2.7 \mathrm{GVm}^{-1}$ for the cubic structure (with $\Delta E_{\mathrm{m}}=0.43 \mathrm{eV}$ [67]) and $\mathcal{E}_{\mathrm{m} \text {,mon }}^{\mathrm{cr}}=4.1 \mathrm{GVm}^{-1}$ for the monoclinic structure (with $\Delta E_{\mathrm{m}}=0.66 \mathrm{eV}$ [67]). The higher migration barrier for oxygen-ion migration in the monoclinic structure allows higher fields to be applied before the structure disintegrates. Second, it was observed that anti-Frenkel pairs are formed sporadically in the monoclinic supercell for fields of $3 \mathrm{GVm}^{-1}$ along the $a$ and $c$ directions, with formation along the $c$ direction preferred over that along the $a$ direction. However, these defect pairs neither separated from each other nor remained stable-despite the extreme fields-but instead recombined within the next few picoseconds $\left(t_{\mathrm{rec}, \mathrm{a}}<1 \mathrm{ps}\right.$ and $\left.t_{\mathrm{rec}, \mathrm{c}}<4 \mathrm{ps}\right)$. In the cubic supercell no defect pairs were formed during the entire course of the simulation at all stable fields $\left[\mathcal{E}=\left(0 \mathrm{GVm}^{-1} ; 3 \mathrm{GVm}^{-1}\right)\right]$.

Our MD results are thus consistent with the prediction of Eq. (6) - that $\mathcal{E} \sim 10^{1} \mathrm{GV} \mathrm{m}^{-1}$ is necessary for the generation of anti-Frenkel pairs - but we are unable to confirm the prediction since the crystals become unstable in our simulations. Nevertheless, this leads us to the conclusion that, for all materials for which $\Delta E_{\mathrm{m}}<\Delta E_{\mathrm{aF}}$ is valid (see Fig. 4), the material will become unstable at some field strength that is much smaller than the critical field required for the generation

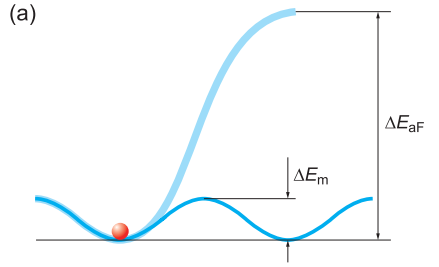

(b)

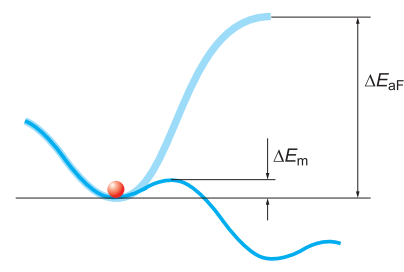

FIG. 4. Schematic comparison of energy profiles for an oxygenion moving over a barrier to a vacant site and to an interstitial site (a) without an applied field and (b) with an applied field. In oxides for which $\Delta E_{\mathrm{m}}<\Delta E_{\mathrm{aF}}$ holds, a superimposed field will distort the migration barrier to zero before anti-Frenkel pairs can form.

of anti-Frenkel pairs. These fields, in any case, are much higher than fields than can be applied in experiment without experiencing problems.

\section{Dynamics of recombination}

The recombination of anti-Frenkel defect pairs was examined separately through MD simulations, with and without an applied field. Specifically, we investigate the case of a doubly charged interstitial $\mathrm{O}_{i}^{\prime \prime}$ and a neutral oxygen vacancy $\mathrm{V}_{\mathrm{O}}^{\times}$, that is, an oxygen vacancy with neighboring hafnium ions which are reduced by two electrons. For the case of a doubly charged interstitial and a doubly charged vacancy the Coulomb interaction is strong enough to force immediate (within a few ps) recombination as shown above and found earlier by Bradley et al. [37] and Clima et al. [64]. The $\left[\mathrm{V}_{\mathrm{O}}^{\times}-\mathrm{O}_{\mathrm{i}}^{\prime \prime}\right]$ pairs are created by the following procedure. First, we introduced a random distribution of oxygen interstitials to the lattice and subsequently relaxed the supercell to its minimum energy in an MD simulation at $T=1000 \mathrm{~K}$ for $100 \mathrm{ps}$. Next, for every interstitial the closest oxygen ion was removed from the lattice to form a doubly positively charged oxygen vacancy and two nearest-neighbor $\mathrm{Hf}^{4+}$ ions were reduced to $\mathrm{Hf}^{3+}$ (ion charges were modified but the short-range potentials remained unchanged) to yield in total a neutral oxygen vacancy. This approach intrinsically produced three-fold and four-fold coordinated oxygen vacancies. Then MD simulations were conducted at $T=1000 \mathrm{~K}$ for another $100 \mathrm{ps}$ in a field of $\left(0 \mathrm{GVm}^{-1} ; 3 \mathrm{GVm}^{-1}\right)$ and the behavior of the anti-Frenkel pairs is tracked every $0.1 \mathrm{ps}$. All defect pairs were observed to recombine within (0.1 ps; $0.6 \mathrm{ps})$ in the cubic phase and within $<0.1 \mathrm{ps}$ in the monoclinic phase, even though nominally there is no Coulomb interaction between them. There are still elastic interactions, however, between vacancy and interstitial moieties; and the Coulomb interaction between vacancy and interstitial may only become negligible at large defect-defect separations, such that the vacancy together with its surroundings appear neutral.

\section{DISCUSSION}

In the previous sections, we presented two approaches: a (simple) thermodynamic model (along with some more complicated additions) and atomistic simulations. Both approaches unambiguously demonstrated that anti-Frenkel 
pairs are extremely unlikely to form under an applied field in $\mathrm{HfO}_{2}$.

Now, one may criticize that the bond-breaking mechanism introduced by McPherson and Mogul [77] is not accounted for in either of these approaches. It is not considered in the thermodynamic model, and in the atomistic simulations, it is debatable to what degree this effect is included: one the one hand, electrons are not specifically included (we use the Born model of solids and the shell model for ion polarizability [78] is neglected); on the other hand, the applied field is superimposed on the interatomic pair potentials. Here we argue that the application of the bond-breaking model to anti-Frenkel pair formation is - that is, the polarization of $\mathrm{Hf}-\mathrm{O}$ bonds by an external electric field and the subsequent destabilization of a single bond in the local field of all other polarized bonds-invalid if derived from the Lorentz equation and the Clausius-Mossotti equation.

Generally, the Clausius-Mossotti equation, and with it the bond-breaking approach, is only valid for nonpolar gases, and in special cases, for a few liquids. In the model case of a cubic array of conducting spheres, it has already been shown to fail by Doyle [79]. Furthermore, Fröhlich [80] pointed out that to derive the Clausius-Mossotti equation, from his more general equation for dielectric crystals, two assumptions must hold. First, the only interaction between neighboring unit cells should be electrostatic; this requires the inequality $\left(k_{\mathrm{B}} T \gg\right.$ short-range interaction) to be true. The typical short-range interaction energies in solids (even in ionic solids, see Table I) are of the order of $\mathrm{eV}$, and hence, extremely high temperatures are required for the inequality to hold. Second, the sphere, within which the local electric field is calculated, should display the same dielectric properties as the macroscopic crystal; this is obviously not the case for crystalline solids because the properties heavily depend on the lattice symmetries and not only on one (unit) cell. A further argument against the bond-breaking mechanism comes from a recent study by Youssef et al. [81]. Using DFT calculations (which of course specifically include electrons and ion polarizability) they determined that the formation energy of a neutral vacancy in $\mathrm{MgO}\left(\Delta G_{\mathrm{V}_{0}^{\times}}^{\text {form }}\right)$ decreases by only $0.5 \mathrm{eV}$ at a field strength of $\mathcal{E}=2.5 \mathrm{GV} \mathrm{m}^{-1}$. Thus, one may suppose that there is an effect on the generation of anti-Frenkel pairs in $\mathrm{HfO}_{2}$, but the field strengths are likely to be huge and the effects are likely to be small.

Finally, we turn our attention to the implications for the two phenomena for which field-enhanced defect generation has been proposed: resistive switching and flash sintering. For the former, in addition to requiring highly unrealistic field strengths, this approach further lacks reversibility, a crucial part of the resistive switching mechanism being the ability to switch between high and low resistance states (HRS and LRS). Let us assume that the applied field has created some antiFrenkel pairs and removed the charged oxygen interstitials, thus forming a conductive filament of oxygen vacancies [see
Eq. (3)] and putting the device into a LRS. If the polarity is now switched, however, further generation of anti-Frenkel pairs will occur: there is a field applied across the oxide and electrons are continually being supplied to neutralize oxygen vacancies. As a consequence, the filament will not be disrupted and the device remains in the LRS, instead of switching back to the HRS.

O'Hara et al. [82] also showed that oxygen-vacancy formation close to an interface with an oxygen reservoir-or, as termed in their work, "Frenkel-Pair formation across the interface"-is favored over formation in the bulk. The oxygen atom is considered to migrate into the reservoir immediately and does not reside as an interstitial in the oxide. Such a reaction is, however, identical to Eq. (1), that is, the removal of an oxygen atom from the oxide layer without any oxygen interstitials being involved. Calling the reduction reaction of Eq. (1) "Frenkel-Pair formation across the interface" is simply a misnomer. Consequently, the exchange of oxygen with a reservoir constitutes, currently, the most plausible mechanism for defect generation in resistive switching.

Flash sintering refers to the sudden densification of a ceramic body in an applied electric field. In the case of $\mathrm{Y}_{2} \mathrm{O}_{3}$-substituted $\mathrm{ZrO}_{2}$, for example, a field strength of only $\mathcal{E}=10^{4} \mathrm{~V} \mathrm{~m}^{-1}$ is sufficient to decrease simultaneously the sintering temperature from $1450{ }^{\circ} \mathrm{C}$ to $850^{\circ} \mathrm{C}$ and the time to full densification from hours to mere seconds [83]. Since oxides such as $\mathrm{ZrO}_{2}$ (or $\mathrm{CeO}_{2}$ or $\mathrm{HfO}_{2}$ ) are characterized by the cations being far less mobile than the anions [84-87], it is the cations that determine the sintering behavior. Hence, if a field-induced mechanism of defect generation were to be operative in flash sintering, it would be the generation of Frenkel pairs, not anti-Frenkel pairs, that would be relevant. Simulations predict energies of Frenkel pair formation in $\mathrm{ZrO}_{2}, \mathrm{CeO}_{2}$, and $\mathrm{HfO}_{2}$ in the region of $10-12 \mathrm{eV}[44,46]$. Consequently, with the analogous version of Eq. (6) for Frenkel pairs, we obtain critical field strengths $\mathcal{E}_{\mathrm{F}}^{\mathrm{cr}} \sim 10^{1} \mathrm{GVm}^{-1}$, six orders of magnitude larger than those used experimentally. We conclude, therefore, that field-induced generation of defects plays no role in the flash sintering of fluorite-structured oxides.

The route to generating anti-Frenkel or Frenkel pairs in an oxide through the application of an applied field is often considered, as we describe above, to be a shortcut. Through our thermodynamic model and our atomistic simulations, we conclude that this route is a dead-end.

\section{ACKNOWLEDGMENTS}

This work was supported by the Deutsche Forschungsgemeinschaft (DFG) within the framework of the collaborative research center "Nanoswitches" (SFB917). The computing time granted by the John von Neumann Institute for Computing (NIC) and provided on the supercomputer JURECA at the Jülich Supercomputing Centre (JSC) (Project ID: JPGI70) is gratefully acknowledged. The authors thank M. Martin for helpful discussions.
[1] J. Frenkel, Z. Phys. 35, 652 (1926).

[2] C. Wagner and W. Schottky, Z. Phys. Chem., Abt. B 11, 163 (1930).
[3] F. A. Kröger and H. J. Vink, Solid State Phys. 3, 307 (1956).

[4] L. Heyne, Electrochim. Acta 15, 1251 (1970).

[5] J. Maier, Angew. Chem., Int. Ed. Engl. 32, 313 (1993). 
[6] H. Schmalzried, Chemical Kinetics of Solids (John Wiley \& Sons, New York, 2008).

[7] H. Matzke, Radiat. Eff. 64, 3 (1982).

[8] N. Itoh and K. Tanimura, Radiat. Eff. 98, 269 (1986).

[9] E. Kotomin and A. Popov, Nucl. Instrum. Methods Phys. Res., Sect. B 141, 1 (1998).

[10] H. Pagnia and N. Sotnik, Phys. Stat. Sol. 108, 11 (1988).

[11] M. N. Kozicki, M. Yun, L. Hilt, and A. Singh, in Proceedings of the International Symposium on Solid-State Ionic Devices, edited by E. D. Wachsman, J. R. Akridge, M. Liu, and N. Yamazoe (Electrochemical Society, Pennington, NJ, USA, 1999), Vol. 99-13, p. 298.

[12] R. Raj, M. Cologna, and J. S. C. Francis, J. Am. Ceram. Soc. 94, 1941 (2011).

[13] J. S. Francis, M. Cologna, and R. Raj, J. Eur. Ceram. Soc. 32, 3129 (2012).

[14] S. Murugavel and B. Roling, J. Non-Cryst. Solids 351, 2819 (2005).

[15] B. Roling, S. Murugavel, A. Heuer, L. Luhning, R. Friedrich, and S. Rothel, Phys. Chem. Chem. Phys. 10, 4211 (2008).

[16] M. Pešic, F. P. G. Fengler, L. Larcher, A. Padovani, T. Schenk, E. D. Grimley, X. Sang, J. M. LeBeau, S. Slesazeck, U. Schroeder, and T. Mikolajick, Adv. Funct. Mater. 26, 4601 (2016).

[17] S. Starschich, S. Menzel, and U. Böttger, J. Appl. Phys. 121, 154102 (2017).

[18] M. Vollmann, R. Hagenbeck, and R. Waser, J. Am. Ceram. Soc. 80, 2301 (1997).

[19] R. A. De Souza, Phys. Chem. Chem. Phys. 11, 9939 (2009).

[20] R. Meyer, A. F. Zurhelle, R. A. De Souza, R. Waser, and F. Gunkel, Phys. Rev. B 94, 115408 (2016).

[21] D. Ielmini and R. Waser, Resistive Switching: From Fundamentals of Nanoionic Redox Processes to Memristive Device Applications (Wiley-VCH, New York, 2016).

[22] R. Waser, R. Dittmann, G. Staikov, and K. Szot, Adv. Mater. 21, 2632 (2009).

[23] G. Bersuker, D. C. Gilmer, D. Veksler, P. Kirsch, L. Vandelli, A. Padovani, L. Larcher, K. McKenna, A. Shluger, V. Iglesias, M. Porti, and M. Nafria, J. Appl. Phys. 110, 124518 (2011).

[24] B. Gao, B. Sun, H. Zhang, L. Liu, X. Liu, R. Han, J. Kang, and B. Yu, IEEE Electron Device Lett. 30, 1326 (2009).

[25] X. Guan, S. Yu, and H. Wong, IEEE Trans. Electron Devices 59, 1172 (2012).

[26] S. Yu, X. Guan, and H. Wong, IEEE Trans. Electron Devices 59, 1183 (2012).

[27] P. Huang, X. Y. Liu, B. Chen, H. T. Li, Y. J. Wang, Y. X. Deng, K. L. Wei, L. Zeng, B. Gao, G. Du, X. Zhang, and J. F. Kang, IEEE Trans. Electron Devices 60, 4090 (2013).

[28] B. Gao, J. F. Kang, Y. S. Chen, F. F. Zhang, B. Chen, P. Huang, L. F. Liu, X. Y. Liu, Y. Y. Wang, X. A. Tran, Z. R. Wang, H. Y. Yu, and A. Chin, in Electron Devices Meeting (IEDM), 2011 IEEE International [Electron Devices Meeting (IEDM), 2011 IEEE International, 2011], pp. 17.4.1-17.4.4.

[29] H.-S. P. Wong, H.-Y. Lee, S. Yu, Y.-S. Chen, Y. Wu, P.-S. Chen, B. Lee, F. T. Chen, and M.-J. Tsai, Proc. IEEE 100, 1951 (2012).

[30] A. Padovani, L. Larcher, O. Pirrotta, L. Vandelli, and G. Bersuker, IEEE Trans. Electron Devices 62, 1998 (2015).

[31] B. Traore, P. Blaise, and B. Sklenard, J. Phys. Chem. C 120, 25023 (2016)
[32] B. Traore, P. Blaise, E. Vianello, L. Perniola, B. D. Salvo, and Y. Nishi, IEEE Trans. Electron Devices 63, 360 (2016).

[33] K. Xiong, J. Robertson, M. C. Gibson, and S. J. Clark, Appl. Phys. Lett. 87, 183505 (2005).

[34] J. L. Gavartin, D. M. Ramo, A. L. Shluger, G. Bersuker, and B. H. Lee, Appl. Phys. Lett. 89, 082908 (2006).

[35] A. S. Foster, A. L. Shluger, and R. M. Nieminen, Phys. Rev. Lett. 89, 225901 (2002).

[36] A. S. Foster, F. Lopez Gejo, A. L. Shluger, and R. M. Nieminen, Phys. Rev. B 65, 174117 (2002).

[37] S. R. Bradley, G. Bersuker, and A. L. Shluger, J. Phys.: Condens. Matter 27, 415401 (2015).

[38] S. R. Bradley, A. L. Shluger, and G. Bersuker, Phys. Rev. Appl. 4, 064008 (2015).

[39] R. Poeppel and J. Blakely, Surf. Sci. 15, 507 (1969).

[40] J. Maier, Solid State Ionics 143, 17 (2001).

[41] C. Zener, Proc. Royal Soc. A 145, 523 (1934).

[42] S. Sze and K. Ng, Physics of Semiconductor Devices (Wiley, New York, 2006).

[43] N. Capron, P. Broqvist, and A. Pasquarello, Appl. Phys. Lett. 91, 192905 (2007).

[44] J. X. Zheng, G. Ceder, T. Maxisch, W. K. Chim, and W. K. Choi, Phys. Rev. B 75, 104112 (2007).

[45] Y. Guo and J. Robertson, Appl. Phys. Lett. 105, 223516 (2014).

[46] T. Zacherle, A. Schriever, R. A. De Souza, and M. Martin, Phys. Rev. B 87, 134104 (2013).

[47] M. W. D. Cooper, M. J. D. Rushton, and R. W. Grimes, J. Phys.: Condens. Matter 26, 105401 (2014).

[48] K. Clausen, W. Hayes, J. E. Macdonald, R. Osborn, and M. T. Hutchings, Phys. Rev. Lett. 52, 1238 (1984).

[49] J.-P. Crocombette, D. Torumba, and A. Chartier, Phys. Rev. B 83, 184107 (2011).

[50] Y. Lu, Y. Yang, and P. Zhang, J. Phys.: Condens. Matter 24, 225801 (2012).

[51] S. Murphy, M. Cooper, and R. Grimes, Solid State Ionics 267, 80 (2014).

[52] T. Diokh, E. Le-Roux, S. Jeannot, P. Candelier, L. Perniola, J. Nodin, V. Jousseaume, T. Cabout, H. Grampei, E. Jalaguier, and B. D. Salvo, 2013 IEEE International Reliability Workshop. Final Report (2013), doi:10.1109/IIRW.2013.6804170.

[53] Y. Y. Chen, L. Goux, L. Pantisano, J. Swerts, C. Adelmann, S. Mertens, V. V. Afanas'ev, X. P. Wang, B. Govoreanu, R. Degraeve, S. Kubicek, V. Paraschiv, B. Verbrugge, N. Jossart, L. Altimime, M. Jurczak, J. Kittl, G. Groeseneken, and D. J. Wouters, Microelectron. Eng. 112, 92 (2013).

[54] P. Jancovic, B. Hudec, E. Dobrocka, J. Derer, J. Fedor, and K. Froehlich, Appl. Surf. Sci. 312, 112 (2014).

[55] F. Puglisi, A. Qafa, and P. Pavan, IEEE Electron Device Lett. 36 , 244 (2015).

[56] F. Puglisi, L. Larcher, A. Padovani, and P. Pavan, IEEE Trans. Electron Devices 62, 2606 (2015).

[57] F. Palumbo, E. Miranda, G. Ghibaudo, and V. Jousseaume, IEEE Electron Device Lett. 33, 1057 (2012).

[58] P. Lorenzi, R. Rao, and F. Irrera, IEEE Trans. Electron Devices 60, 438 (2013).

[59] S. Lee, W.-G. Kim, S.-W. Rhee, and K. Yong, J. Electrochem. Soc 155, H92 (2008).

[60] J. J. O'Dwyer, The Theory of Electrical Conduction and Breakdown in Solid Dielectrics (Clarendon, New York, 1973). 
[61] S. Grieshammer, T. Zacherle, and M. Martin, Phys. Chem. Chem. Phys. 15, 15935 (2013).

[62] A. R. Genreith-Schriever and R. A. De Souza, Phys. Rev. B 94, 224304 (2016).

[63] A. Walsh, S. M. Woodley, C. R. A. Catlow, and A. A. Sokol, Solid State Ionics 184, 52 (2011).

[64] S. Clima, B. Govoreanu, M. Jurczak, and G. Pourtois, Microelectron. Eng. 120, 13 (2014).

[65] L. Onsager, J. Chem. Phys. 2, 599 (1934).

[66] C. L. Braun, J. Chem. Phys. 80, 4157 (1984).

[67] M. Schie, M. P. Müller, M. Salinga, R. Waser, and R. A. De Souza, J. Chem. Phys. 146, 94508 (2017).

[68] M. P. Müller and R. A. De Souza, Appl. Phys. Lett. 112, 051908 (2018).

[69] J. E. Jaffe, R. A. Bachorz, and M. Gutowski, Phys. Rev. B 72, 144107 (2005).

[70] J. D. Gale and A. L. Rohl, Mol. Simul. 29, 291 (2003).

[71] G. V. Lewis and C. R. A. Catlow, J. Phys. C: Solid State Phys. 18, 1149 (1985).

[72] W. C. Swope, H. C. Andersen, P. H. Berens, and K. R. Wilson, J. Chem. Phys. 76, 637 (1982).

[73] G. J. Martyna, M. E. Tuckerman, D. J. Tobias, and M. L. Klein, Mol. Phys. 87, 1117 (1996).

[74] I. T. Todorov, W. Smith, K. Trachenko, and M. T. Dove, J. Mater. Chem. 16, 1911 (2006).
[75] S. P. Waldow and R. A. De Souza, ACS Appl. Mater. Interfaces 8, 12246 (2016).

[76] N. F. Mott and M. J. Littleton, Trans. Faraday Soc. 34, 485 (1938).

[77] J. McPherson and H. Mogul, J. Appl. Phys. 84, 1513 (1998).

[78] B. G. Dick and A. W. Overhauser, Phys. Rev. 112, 90 (1958).

[79] W. T. Doyle, J. Appl. Phys. 49, 795 (1978).

[80] H. Fröhlich, Trans. Faraday Soc. 44, 238 (1948).

[81] M. Youssef, K. J. Van Vliet, and B. Yildiz, Phys. Rev. Lett. 119, 126002 (2017).

[82] A. O’Hara, G. Bersuker, and A. A. Demkov, J. Appl. Phys. 115, 183703 (2014).

[83] M. Cologna, B. Rashkova, and R. Raj, J. Am. Ceram. Soc. 93, 3556 (2010).

[84] M. Kilo, M. A. Taylor, C. Argirusis, G. Borchardt, B. Lesage, S. Weber, S. Scherrer, H. Scherrer, M. Schroeder, and M. Martin, J. Appl. Phys. 94, 7547 (2003).

[85] M. Kilo, G. Borchardt, B. Lesage, O. Kaïtasov, S. Weber, and S. Scherrer, J. Eur. Ceram. Soc. 20, 2069 (2000).

[86] S. Beschnitt, T. Zacherle, and R. A. De Souza, J. Phys. Chem. C 119, 27307 (2015).

[87] S. Beschnitt and R. A. De Souza, Solid State Ionics 305, 23 (2017). 